\title{
Duplicación intestinal: Diagnóstico y tratamiento de una condición inusual*
}

\author{
Drs. ALEJANDRA LETELIER M. ${ }^{1}$, CARLOS BARRÍA M. ${ }^{2}$, MARCELO A. BELTRÁN S. ${ }^{3}$, CELIA MORENO CH ${ }^{1}$
}

Servicio de Cirugía Infantil, Hospital de La Serena.

Histomed-La Serena.

3 Servicio de Cirugía, Hospital de La Serena.

Chile.

\begin{abstract}
Intestinal duplication: Diagnosis and treatment of an unusual condition

We report a 2.5 years old male presenting with abdominal pain, nausea and vomiting lasting 30 days. A CAT scan showed an intestinal duplication cyst. The patient was operated and a cystic lesion of $6 \mathrm{~cm}$ diameter was fond and excised along with five $\mathrm{cm}$ of terminal ileum. Intestinal transit was reconstituted with a terminal anastomosis between ileum and colon.
\end{abstract}

Key words: Intestinal duplication, intestinal cyst, ileal excision.

\section{Resumen}

Reportamos el caso clínico de un paciente de 2 años y 6 meses de edad que fue intervenido quirúrgicamente por una duplicación intestinal del ileon terminal. Discutimos la presentación clínica, estudio radiológico, procedimiento quirúrgico y resultados del tratamiento. Revisamos la literatura actual pertinente haciendo énfasis en el diagnóstico y tratamiento de estas malformaciones por el potencial de malignidad que presentan en la vida adulta.

Palabras clave: Duplicación intestinal, malformación intestinal congénita, tumores del ileon.

\section{Introducción}

Las duplicaciones intestinales son anomalías congénitas que se presentan con una frecuencia de $0,2 \%$ en niños ${ }^{1}$. Estas malformaciones se han descrito a lo largo de todo el tracto digestivo, desde la base de la lengua hasta el recto ${ }^{1-4}$. La localización más frecuente de las duplicaciones es el ileon (más del $40 \%$ de los casos), y con menor frecuencia se encuentran en esófago, colon, yeyuno, estómago, duodeno y recto ${ }^{1-7}$. Habitualmente se presentan como duplicaciones únicas, pero pueden ser múltiples hasta en el $15 \%$ de los casos ${ }^{1}$.

La clínica de las duplicaciones intestinales es inespecífica, presentándose con náusea, vómito, dolor abdominal recurrente, presencia de masa abdominal, hemorragia digestiva, perforación y obstrucción intestinal ${ }^{2}$. El diagnóstico preoperatorio de las duplicaciones intestinales hasta una época reciente fue relativamente infrecuente. Actualmente,

*Recibido el 17 de Julio de 2008 y aceptado para publicación el 15 de Septiembre de 2008.

Correspondencia: Dr. Marcelo A. Beltrán S.

Casilla 912. La Serena, IV Región. Chile

E-mail: beltran_01@yahoo.com 
sin embargo, y debido a la amplia disponibilidad de estudios radiológicos, el diagnóstico preoperatorio es habitual ${ }^{2,7}$. El tratamiento quirúrgico consiste en la resección de la lesión y el intestino adyacente seguido de una anastomosis primaria ${ }^{1,2,8,9}$. En el presente reporte discutimos un caso de duplicación intestinal quística del ileon terminal, la cual fue diagnosticada en el preoperatorio, y revisamos la literatura pertinente.

\section{Reporte de caso}

Paciente masculino de 2 años y 6 meses de edad, hospitalizado por un cuadro clínico de 30 días de evolución caracterizado por dolor abdominal asociado a náusea y vómito. El estudio diagnóstico consistió en una ecografía abdominal la cual informa una imagen quística ovalada en la región periumbilical y flanco derecho, que mide 63 x 40 x $35 \mathrm{~mm}$ de diámetro, cuyo contenido es anecogénico y el que está delimitada por una pared con una capa ecogénica y una capa hipoecogénica con refuerzo acústico posterior. La lesión descrita es interpretada como un quiste de duplicación intestinal por el radiólogo.

Con este estudio el paciente se hospitaliza y durante la hospitalización se concluye el estudio con exámenes de sangre habituales, los que son normales, y con una tomografía abdominal computarizada (TAC) con contraste endovenoso, la cual describe una imagen hipodensa de aspecto quístico con contenido líquido espeso, de contornos bien definidos y delimitada por una pared fina sin tabiques internos, que se encuentra en íntimo contacto con la pared anterior del colon descendente y ciego (Figura 1), sin infiltrar los órganos o tejidos vecinos. En sus ejes longitudinal y transverso mide 56 x 39 $\mathrm{mm}$. No se identifican otras lesiones. El diagnóstico radiológico es el de un quiste de duplicación entérico.

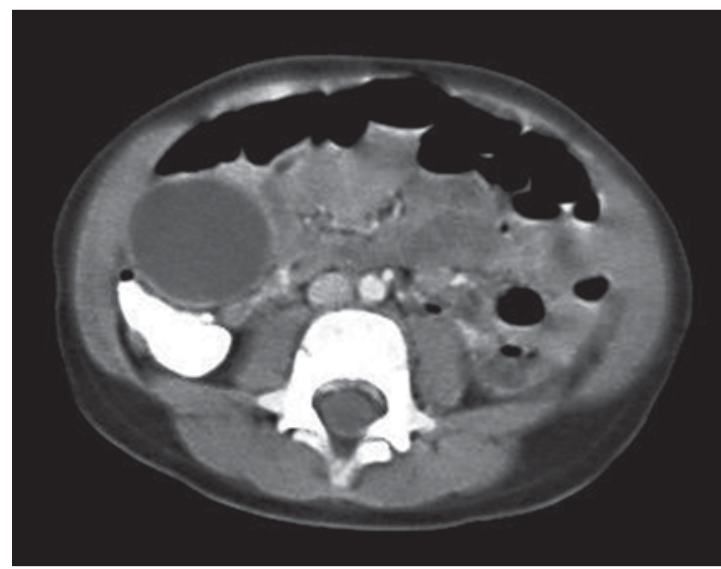

Figura 1.

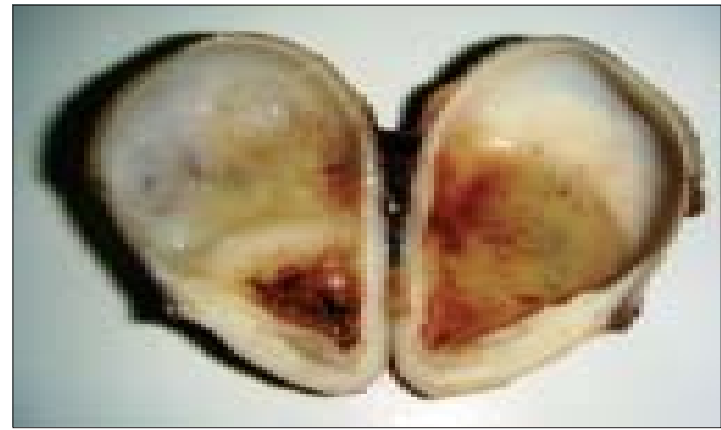

Figura 3.

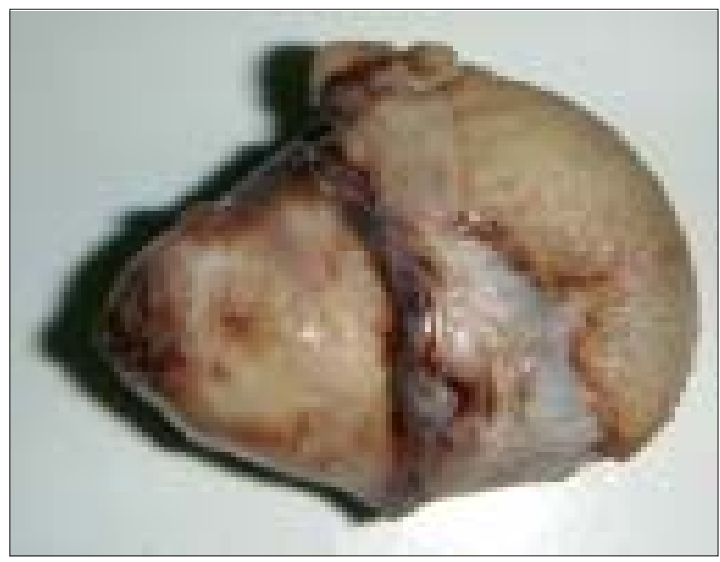

Figura 2.

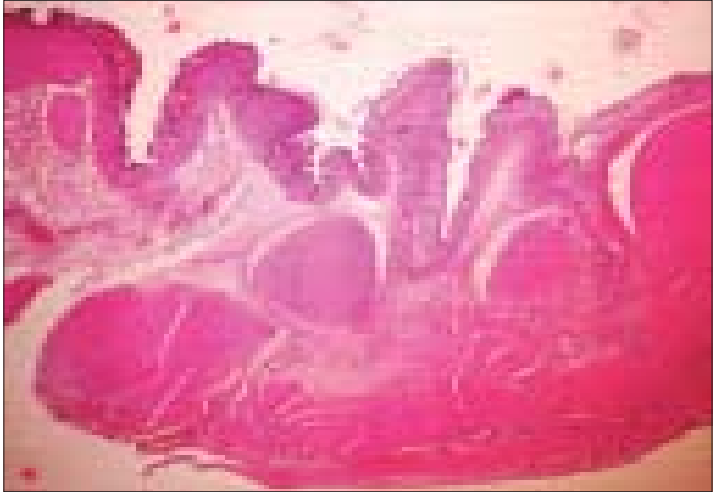

Figura 4. 
En la operación se identifica la tumoración quística en la zona de la válvula ileocecal de aproximadamente $6 \mathrm{~cm}$ de diámetro, la que es extirpada en bloque junto con $5 \mathrm{~cm}$ de ileón terminal, ciego y 5 $\mathrm{cm}$ de colon ascendente. Se reconstruye él transito con una anastomosis término-terminal entre el ileon y el colon en un solo plano. El estudio anatómico y patológico de la pieza quirúrgica reporta una formación quística con una superficie externa parcialmente lisa y parcialmente granulosa (Figura 2), la superficie interna es lisa y brillante y contiene mucus (Figura 3).

Histológicamente el quiste está constituido por músculo liso fasciculado ordenado en capas longitudinales y lisas, su superficie interna se encuentra parcialmente cubierta por epitelio intestinal bien diferenciado aunque las vellosidades se encuentran atróficas, en buena parte de la superficie interna la mucosa no es visible por atrofia, y externamente la pared esta cubierta por serosa (Figura 4).

\section{Discusión}

En 1884 Reginald Fitz introdujo el término "duplicación intestinal" para describir y explicar las anomalías quísticas congénitas del tracto gastrointestinal, las que él pensó que eran remanentes del conducto onfalomesentérico ${ }^{5}$. La mayoría de las publicaciones sobre duplicaciones intestinales constituían entonces reportes de casos clínicos, en los que se utilizaban diversos términos para referirse a estas anomalías. En 1937 Ladd recomendó el uso del término "duplicaciones del tracto alimentario", y las observaciones de este autor consolidaron la clasificación de esta entidad y clarificaron su diferenciación de otras malformaciones quísticas del tracto gastrointestinal ${ }^{10}$. Posteriormente, Gross y Holcomb en 1952, con la intención de simplificar la nomenclatura retrocedieron hasta los trabajos de Fitz, y propusieron que él término "duplicación intestinal" fuera utilizado para todas estas anomalías independientemente de su sitio de origen, morfología o derivación embriológica ${ }^{6}$. Esta nomenclatura es la que actualmente continúa en uso.

Muchas teorías se han propuesto sobre el origen embriológico de las duplicaciones intestinales, ninguna de ellas absolutamente conclusiva. A pesar de que algunas teorías explican el desarrollo de las duplicaciones en diferentes segmentos específicos del tracto digestivo, ninguna puede explicar satisfactoriamente el origen de todas las duplicaciones. Clásicamente se describe la teoría del "gemelo abortivo" que explicaría las duplicaciones del colon asociadas a duplicaciones de estructuras genitales y urinarias ${ }^{11}$. La teoría del "divertículo embriológico persistente" sugiere que pequeños divertículos habitualmente transitorios que se encuentran en el lado antimesentérico de la pared intestinal en el embrión, persisten y se desarrollan en duplicaciones intestinales; sin embargo, la mayoría de las duplicaciones se encuentran en el lado mesentérico del intestino por lo que esta teoría no es fácilmente justificable ${ }^{12}$. Una teoría similar explica satisfactoriamente y asocia entre sí las malformaciones broncopulmonares y esofágicas; sin embargo, no es útil para explicar las duplicaciones intestinales en el resto del tracto digestivo ${ }^{13}$. Una de las teorías más populares es la denominada "teoría de la recanalización luminal aberrante" que explica adecuadamente las duplicaciones de aquellas áreas del tracto digestivo que pasan por una fase sólida como el esófago, intestino delgado y colon ${ }^{14}$.

Actualmente, la teoría más aceptada para las duplicaciones intestinales, principalmente las del intestino delgado, es la del "accidente vascular intrauterino", la cual postula que estas anomalías se producen como resultado de accidentes vasculares intrauterinos ${ }^{15}$. Esta gran variedad de explicaciones, sin una teoría completamente aceptada o demostrada, sugiere que el origen de las duplicaciones intestinales puede ser multifactorial y que la investigación genética y molecular actual probablemente nos brindará una explicación aceptable respecto a estas anomalías en el futuro próximo.

Las manifestaciones clínicas de las duplicaciones intestinales son muy inespecíficas y dependen del tipo de duplicación, de su localización, de la presencia de mucosa gástrica heterotópica y de las complicaciones que pudiesen ocasionar. Los síntomas descritos con mayor frecuencia son el dolor abdominal recurrente, náuseas y vómitos, presencia de masa abdominal y sintomatología asociada a las complicaciones como hemorragia digestiva, obstrucción intestinal y perforación libre hacia el peritoneo. La hemorragia digestiva es una complicación frecuente que se presenta en $34 \%$ de los casos y se debe a la ulceración de la mucosa por la producción de ácido por mucosa gástrica heterotópica o por la isquemia secundaria a compresión extrínseca del intestino normal por una duplicación quística adyacente. La obstrucción intestinal se debe a invaginación, vólvulo intestinal o compresión extrínseca por una duplicación quística. La invaginación intestinal por esta causa se presenta característicamente en el período neonatal. La perforación intestinal es bastante infrecuente y se manifiesta como peritonitis ${ }^{1,2,-9}$. Habitualmente las duplicaciones intestinales se presentan, diagnostican y resuelven en los primeros años de la vida, la mayoría de ellas (72\%) antes de los 2 primeros años 
de vida ${ }^{1,2,7-9,15,16}$, por esta razón la clínica de estas anomalías depende mucho de la edad de presentación y no sólo de las otras características ya descritas. Nuestro paciente de 2 años y 6 meses de edad se presentó con un cuadro clínico absolutamente inespecífico y el diagnóstico de su problema fue posible sólo por medios radiológicos. Se dice que el diagnóstico preoperatorio de las duplicaciones intestinales es infrecuente ${ }^{8,9,17-20}$, sin embargo, esta opinión depende del período histórico de las publicaciones. Actualmente con la amplia disponibilidad de estudios radiológicos como la ecografía, tomografía o resonancia magnética abdomi-

Tabla 1. Clasificación de las duplicaciones intestinales

\begin{tabular}{ll}
\hline Tipo & Descripción de la duplicación \\
\hline 1 & Extramesentérica \\
1A & Meso independiente del meso intestinal \\
1B & Meso común con el meso intestinal \\
1C & Duplicación unida al intestino por una pared \\
& muscular común \\
2 & Intramesentérica \\
2A & Pared de la duplicación separada de la pared \\
& intestinal \\
2B & Duplicación unida al intestino por una pared \\
& muscular común \\
\hline
\end{tabular}

nal, el diagnóstico preoperatorio de las duplicaciones intestinales en la infancia no es infrecuente ${ }^{1,7,8}$. Como ocurrió en este caso, el radiólogo puede sospechar esta anomalía basándose en sus características ecográficas: masa quística con una capa mucosa interna ecogénica y una capa muscular externa hipoecogénica ${ }^{7}$. Para confirmar este diagnóstico puede utilizarse la tomografía o resonancia magnética abdominal; en estos estudios las duplicaciones aparecerán como estructuras quísticas redondeadas llenas de líquido o como estructuras tubulares de paredes delgadas adyacentes a las paredes de los intestinos ${ }^{7,8}$.

El año 1998; Long et $\mathrm{al}^{16}$, clasificaron las duplicaciones intestinales de acuerdo al tipo de irrigación de la malformación y su relación con el intestino delgado y su mesenterio (Tabla 1). Las duplicaciones intestinales Tipo 1 son las que se encuentran a un lado del mesenterio y las arterias que las irrigan transcurren paralelas e independientes a la vascularización intestinal. Las duplicaciones intestinales Tipo 2, son las que se encuentran en el medio de ambas cubiertas peritoneales del mesenterio y su irrigación proviene de las arterias que irrigan el intestino y que rodean ambas superficies de la duplicación (Figura 5).

De acuerdo a esta clasificación, la duplicación intestinal de nuestro paciente corresponde a una duplicación quística tipo $1 \mathrm{~A}$ en la cual la malformación presenta un mesenterio vascular propio, la cual, junto con el subtipo $1 \mathrm{~B}$, pueden ser completamente

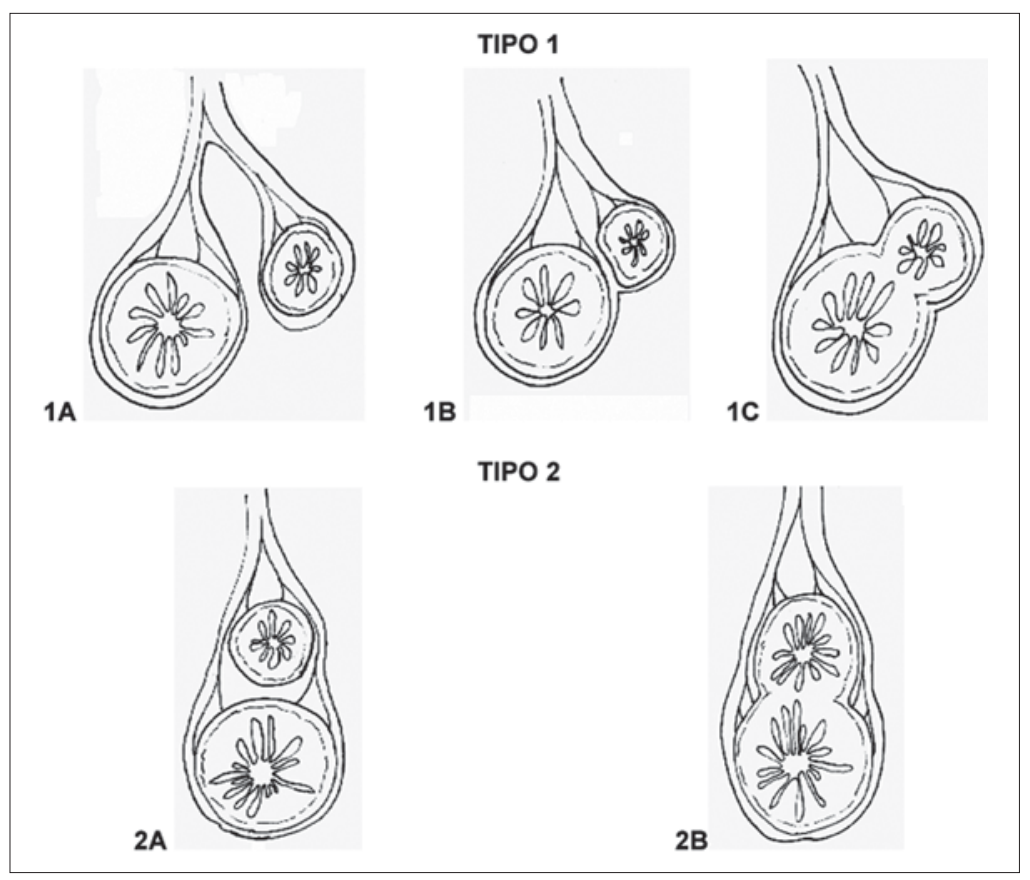

Figura 5. Clasificación de las duplicaciones intestinales según Long et $\mathrm{al}^{16}$. 
resecadas, tal como sucedió en este caso. La resección completa de la duplicación no es habitualmente posible en los otros subtipos, por lo que el reconocimiento apropiado del subtipo de duplicación es fundamental para planificar la estrategia quirúrgica. El tratamiento quirúrgico de las duplicaciones intestinales debe estar dirigido a la completa resección de toda la malformación y el intestino delgado adyacente comprometido; esto es habitualmente posible reconstituyendo después la anatomía con anastomosis término-terminales ${ }^{8,9,17-20}$.

En nuestro paciente la resección completa de la duplicación quística junto con el intestino asociado (ileon terminal y ciego) fue posible y la reconstitución con una anastomosis término-terminal entre el ileon distal y el colon ascendente no representó un problema. En adultos se han reportado 15 casos de duplicaciones intestinales del ileon tratadas quirúrgicamente, de ellos en 4 casos (28\%) la duplicación degeneró en un adenocarcinoma, hecho que demuestra la tendencia a la malignización de este epitelio excluido y el potencial peligro de estas anomalías cuando no son tratadas quirúrgicamente en la infancia ${ }^{21-24}$.

Las duplicaciones intestinales son anomalías infrecuentes del desarrollo embriológico del tracto gastrointestinal y se presentan habitualmente en la infancia. El diagnóstico puede presentar algunas dificultades; sin embargo, los estudios radiológicos confirman el diagnóstico preoperatorio y el tratamiento quirúrgico es simple. Las duplicaciones intestinales que se diagnostican en la infancia deben ser siempre tratadas quirúrgicamente por el potencial de malignización que presentan en la edad adulta.

\section{Referencias}

1. Seguel FR, Alvarez MBQ, Ollero JCF, Rollan VV. Duplicación intestinal independiente. Cir Pediatr 2002; 15: $127-129$.

2. Soares-Oliveira M, Castañón M, Carvalho JL, Ribo JM, Bello P, Estevao-Costa J, et al. Duplicaciones intestinales: Análisis de 18 casos. An Esp Pediatr 2002; 56: $430-433$.

3. Pintér AB, Shubert W, Szemlédy F, Göbel P, Schäfer J, Kustos G. Alimentary tract duplications in infants and children. Eur J Pediatr Surg 1992; 2: 8-12.

4. Chandramouli P, Iyer CP, Mahour GH. Duplications of the alimentary tract in infants and children. J Pediatr Surg 1995; 30: 1267-1270.

5. Fitz RH. Persistent omphalo-mesenteric remains: Their importance in the causation of intestinal duplication, cyst formation, and obstruction. Am J Med Sci 1884;
88: $30-57$.

6. Gross RE, Holcomb GW, Farber S. Duplications of the alimentary tract. Pediatrics 1952; 9: 449-468.

7. Macpherson RI. Gastrointestinal tract duplications: Clinical, pathologic, etiologic, and radiologic considerations. RadioGraphics 1993; 13: 1063-1080.

8. Holcomb GW, Gheissari A, O’Neill JA, Shorter NA, Bishop HC. Surgical management of alimentary tract duplications. Ann Surg 1989; 209:.167-174.

9. Ildstad ST, Tollerud DJ, Weiss RG, Ryan DP, McGowan MA, Martin LW. Duplications of the alimentary tract: Clinical characteristics, preferred treatment, and associated malformations. Ann Surg 1988; 208: 184-189.

10. Ladd WE. Duplications of the alimentary tract. South Med J 1937; 30: 363.

11. Edwards H. Congenital diverticula of the intestine: With report of a case exhibiting heterotopia. $\mathrm{Br} \mathrm{J}$ Surg 1929; 17:.7-21.

12. Lewis FT, Thyng FW. The regular occurrence of intestinal diverticula in embryos of the pig, rabbit, and man. Am J Anat 1908; 7: 505-519.

13. Heithoff KB, Sane SM, Williams HJ, Jarvis CJ, Carter J, Kane P. Bronchopulmonary-foregut malformations: A unifying etiologic concept. Am J Radiol 1976; 126: 46-55.

14. Bremer JL. Diverticula and duplications of the intestinal tract. Arch Pathol 1944; 38: 132-140.

15. Favara BE, Franciosi RA, Akers DR. Enteric duplications: Thirty-seven cases: A vascular theory of pathogenesis. Am J Dis Child 1971; 122: 501-506.

16. Long L, Zhang JZ, Wang YX. Vascular classification for small intestinal duplications: Experience with 80 cases. J Pediatr Surg 1998; 33: 1243-1245.

17. Bower RJ, Sieber WK, Kiesewetter WB. Alimentary tract duplications in children. Ann Surg 1978; 188: 669-674.

18. Webb JRD. Two cases of duplication of the alimentary canal. Proc Royal Soc Med 1950; 43: 613-614.

19. Fecher MP, Day SM, King FG. Ileal duplications. Ann Surg 1952; 135: 555-559.

20. Hardaway RM, Wedgwood RJ, Swartley RN, Rudman I. Duplication of the terminal ileum. Ann Surg 1952; 136: 296-298.

21. Devos B, Schreurs L, Duponselle E, Hendrix T, Van Dijck H, Van Vuchelen J. Adenocarcinoma in a cystic duplication of the Ileum. Acta Chir Belg 1987; 87: 235-238.

22. Ribaux C, Meyer P. Adenocarcinoma in an ileal duplication. Ann Pathol 1995; 15: 443-445.

23. Tew K, Soans BK, Millar EA. Adenocarcinoma in an ileal duplications cyst: Ultrasound and tomography findings. Australas Radiol 2000; 44: 228-231.

24. Babu MS, Raza M. Adenocarcinoma in an ileal duplication. J Assoc Physicians India 2008; 56: 119-120. 